\title{
El espacio arquitectónico topológico digital
}

The digital topological architectural space

\author{
Adriana Edith Granero \\ Universidad de Belgrano, Argentina \\ Adriana.granero@gmail.com
}

\begin{abstract}
This present assay is a personal research the author to found the process of morphogenesis of architectural space at the theoretical level. It follows earlier work that the current space demands require experiences that only you can answer the limit of a treaty as an architectural surface topological space. The space today is influenced by the concept of convergence, connectivity, continuity, neighborhood, etc.
\end{abstract}

Keywords: Tetradimensional; Topológico; Diferencial; Morfogénesis Biyectiva

\section{Introducción}

Esta investigación es la evolución de una experiencia previa, dicha acción está vinculada al diseño de superficies construídas como límites de espacios arquitectónicos de grandes luces, para ello se partió de una estructura celular, inspirada en la biomimética. En la búsqueda de perfeccionar una propuesta académica el investigador continuó con el desarrollo de la exploración, pero a nivel teórico de una oferta didáctica del taller de forma y comunicación. La propuesta fue complejizada para llegar al concepto vinculado a los EA (Algoritmos Evolutivos) desde la representación y no desde el análisis. En este documento se expresan los fundamentos de la indagación supuesta, la formulación y la invocación de teorías pertenecientes a otras áreas del conocimiento. El objetivo de estas líneas es exponer los pasos y fundamentos de la experiencia y su transposición didáctica, su vinculación inicial con la biomimética, los procesos que generaron las primeras superficies complejas, con algoritmos evolutivos representados gráfica y materialmente, la relación con la adaptación de unidades o células de la estructura celular propuesta y la transferencia a métodos, técnicas y herramientas de producción asociadas a la enseñanza aprendizaje de la Arquitectura actual, específicamente en el diseño de las envolventes arquitectónicas.

\section{Reflexiones previas}

Hace unos meses hemos tenido acceso a una entrevista realizada por el Arq. Julio Arroyo al Arq. Peter Eisenman publicada en YouTube (Julio Arroyo, 2015), en ella se planteaban diversos problemas vinculados a la enseñanza de la arquitectura y los medios de comunicación o medios digitales. Uno de los primeros problemas señalados en dicha entrevista por el Arq. Eisenman, fue la abrumadora demanda de información que provocan los medios de comunicación actuales, que son muy diferentes a la comunicación de la arquitectura, "La idea y la construcción, llevan tiempo!", "Los medios de comunicación no pueden esperar, necesitan tener algo... una imagen... inmediatamente", "devoran la arquitectura antes que la arquitectura pueda inclusive suceder y necesitan a cada hora un nuevo héroe"... El Arq. Eisenman hace un llamado a la falta de atención por problemas de capacidad o de voluntad. En su discurso deja entrever, que es necesaria una arquitectura que demande mucha atención, como estímulo a una forma de vida contemplativa, donde la arquitectura no dependa de los materiales, ni de las maneras de producción, sino del espacio y las sensaciones que en él, el hombre sea capáz de experimentar en estado de contemplación. Agregando en su relato que por eso Alberti es tan importante en la educación del futuro arquitecto, porque fue él fue el primero en hablar del espacio arquitectónico. En referencia a la construcción del espacio y sus límites, podemos afirmar que la arquitectura estuvo y está influenciada siempre por los cambios tecnológicos y filosóficos, los descubrimientos y las teorías, los avances en otras ciencias, entre otros como agentes atractores. Es por esto que el espacio arquitectónico y la envolvente de ese espacio arquitectónico pueden ser entendidas comos filtros o barreras, que generan y protegen las emociones y se vinculan a la actividad humana.

\section{Enseñanza-aprendizaje de la forma o envolvente del espacio arquitectónico en la era pos-digital y su comunicación}

El desarrollo de la forma de la arquitectura actual parece encontrarse frente a desafíos disentidos; por un lado los requerimientos de menor impacto ambiental y por otro la irrupción de nuevas tecnologías, estos retos tensionan especialmente la formación profesional. Ya sea porque el desafío de un futuro sostenible en el sector de la construcción se ha centrado en reducir el consumo energético y la huella de carbono, lo que implica considerar diversos análisis numéricos del comportamiento del diseño arquitectónico, aparentemente dejando a un lado los aspectos morfológicos de la envolvente de los espacios arquitectónicos. Esta preocupación implica abordar consideraciones abstractas y extensas en relación a la forma de las envolventes, como la evolución de condiciones ambientales o el consumo de calorías en el tiempo y que se relacionan lejanamente con los aspectos formales y espaciales de la forma de la envolvente del espacio arquitectónico y de la arquitectura propiamente dicha, pero directamente trabajados en el ejercicio de la profesión. Este planteo dista del solo hecho de representar gráficamente la arquitectura, a través de la geometría los elementos de arquitectura (que se relacionan con las 
habilidades de comunicación, los conocimientos técnicos de expresión y las herramientas); como así también dista de la representación de datos con símbolos numéricos, algoritmos y gráficos de barras, tortas, curvas, etc. (que se relacionan con los conocimientos técnicos). La dificultad de integrar estos conocimientos técnicos, la creciente disponibilidad y utilización de los nuevos medios de información y comunicaciones, que se caracterizan por su interacción gráfica, con un lenguaje simbólico que se fundamenta en sintagmas o grupos de palabras construyen un escenario distinto. En esta atmósfera, se entiende que es responsabilidad de los profesores o tutores incluir el uso de la Tecnología de Información y Comunicación fundamentada en conceptos relacionados a los cambios filosóficos, los descubrimientos, las nuevas teorías, como los avances en otras ciencias entre otros. Mientras la enseñanza arquitectónica está basada sustancialmente en el diseño de proyectos, la comunicación de la enseñanza y la comunicación de los resultados del aprendizaje se sustentan en la visualización del diseño del proyecto propuesto y en el discurso, de modo que estas posibilidades tecnológicas se constituyen en oportunidades. La didáctica pos-digital expone una estrategia de integración, basada en la experimentación y desarrollo sucesivo y la selección como un mecanismo eficaz de aprendizaje significativo de arquitectura sostenible y la optimización de recursos con impacto ambiental. Los estudios de percepción han comprobado extensamente que los procesos cognitivos desarrollados durante la aprehensión del entorno, validan el predominio visual pero también la permanente relación con los restantes sentidos; además de la memoria y la reflexión. Reconociendo una actividad constante de conexión y configuración mental de las percepciones, vinculadas a la experiencia individual, como también a sus inferencias. Decantándose en una comprensión global de la realidad (Gibson, 1986), o en el procesamiento de la información percibida según la teoría computacional de Marr. De manera similar en las que las teorías educacionales ha esclarecido la diferencia entre la aprehensión memorística y temporal, de la comprensión perdurable, que se identifica como "aprendizaje significativo" (Ausubel et al, 1983). Este proceso se caracteriza, más que por sus medios o contenidos, por establecer relaciones con conocimientos previos del individuo y asociarlo con otras informaciones, así como por su capacidad de reflexionar (metacognición) o por valorar emocionalmente la experiencia, generando modificaciones perdurables en el desarrollo personal. Estas condiciones cognitivas nos permiten comprender fundamentalmente, la acelerada adopción de tecnologías digitales por los jóvenes en etapas de aprendizaje, en que prevalecen los recursos caracterizados por la facilidad de utilización a través de metáforas gráficas y/o por la generación de relaciones afectivas (como los teléfonos móviles o las redes sociales).

\section{La evolución de las herramientas de representación y su influencia en la producción arquitectónica}

La producción arquitectónica académica ha experimentando en los últimos años una evolución, estimulada por el diálogo entre la morfología y la fabricación digital (Muñoz, 2013). Los medios digitales y su aplicación a la modelación y fabricación de maquetas arquitectónicas, permiten la exploración de alternativas con flexibilidad reflexiva, en una producción que nos acerca al espacio relativo, de la mano de los avances vinculados a la materia y a la tecnología. Dalí expresaba en estas palabras que la Arquitectura tiene una dependencia directa con una cuestión técnica y que es solo tener la técnica necesaria para repensar y concretar los proyectos arquitectónicos innovadores (Estévez, 2015). Peter Eisenman decía que lo "clásico" era un sistema de relaciones entre representación, razón e historia y que a pesar de la proclamadas rupturas ideológicas y estilistas de movimientos posteriores al clásico, está relación no había sido cuestionada. Agrega que desde el siglo XV la arquitectura pretende ser un paradigma de lo eterno, significativo y verdadero. Expone que cuando no hay distinción entre la representación y la realidad, cuando la realidad es una simulación y que es en ese instante, cuando la representación pierde su fuente de significado y se transforma en un simulacro. La transformación de la razón desde un origen ideal que conducía a un fin ideal, pasó por el proceso de racionalidad pura, luego por un proceso de funcionalidad pura, atravesó por un proceso técnocrático hasta la red de argumentos preñados de valor que dependen de otra teleología, en el caso de esta experiencia de la que se relaciona con la eficacia biológica. La historia y su relación con el tiempo, que vincula la relación entre los elementos, las culturas y la adecuación, fuertemente relacionados conceptualmente con conceptos de selección natural. John y Julia Frazer, investigaron los fundamentos que crean los procesos de la forma en la arquitectura, teniendo en cuenta la arquitectura como una forma de vida artificial, y proponen una representación genética en una forma de ADN como código de script, que luego puede ser objeto de procesos de desarrollo y la evolución es de respuesta al usuario y al medio ambiente. Dollens (2007) dice "what can be draw, can be built", lo que puedo dibujar, puedo construir (Estévez, 2015). Bueno, en su trabajo de investigación "Consideraciones y recursos para la concepción de la forma en la forma de la arquitectura en la era digital" (Bueno, 2008), retoma el tema biológico y establece criterios con una relación hereditaria y recurrente que genera una superficie compleja, volviendo a vincular conceptos relacionados con las teorías de la evolución y selección natural. Schumacher (Schumacher, 2011) da un paso más adelante en sus investigaciones y designa a la arquitectura como un Sistema capaz de reproducirse y mantenerse por sí mismo. Esta declaración se interpreta como un cambio radical en la forma de concebir la arquitectura, Schumacher esboza que ya no depende de un diseñador externo para la construcción y el mantenimiento, sino de un diseñador principal que sea capaz de generar una estructura profunda, primitiva y simple a partir de la cual se puede realizar modificaciones u operaciones, donde la regla y el principio de combinación relaciona esta estructura profunda, primitiva y simple, con un sistema de pensamiento biológico y de actuación de acuerdo al mismo. El diseñador secundario es el mismo sistema, que a partir del diseño inicial es capaz de rediseñarse y ponerse a prueba en múltiples oportunidades, este rediseño emula procesos de selección natural. Janine Benyus (BENYUS, 2002) describe tres niveles de integración con la biomimética, el primero es el que imita la forma natural, el segundo es el que imita los procesos naturales y el tercero es el que copia funcionamiento de los sistemas, sus conductas. Específicamente a esto se refiere Schumacher cuando habla de la "autopoiesis de la Arquitectura". Según el sistemismo 
(Bunge, 2012) la arquitectura puede ser interpretada como un sistema material físico, que combina otros sistemas materiales y físicos, que contienen a su vez figura(forma). Para Bunge, un sistema está compuesto por unidades que se relacionan con al menos otra unidad, es un objeto complejo, tiene una estructura propia y un límite que lo separa de su entorno. Este sistema está compuesto por elementos de arquitectura, afectados con enlaces físicos y químicos o reglas de composición, que los relacionan de manera estática, pero con defectos por no poseer mecanismos. Entendemos que lo que ha cambiado es el cuarto componente: el mecanismo, este debe permitir un ligamento dinámico. Este concepto se refiere a procesos que se dan dentro del sistema y que provocan un cambio en él y es a lo que se refiere en la entrevista Schumacher con "reproducirse y mantenerse por si mismo".
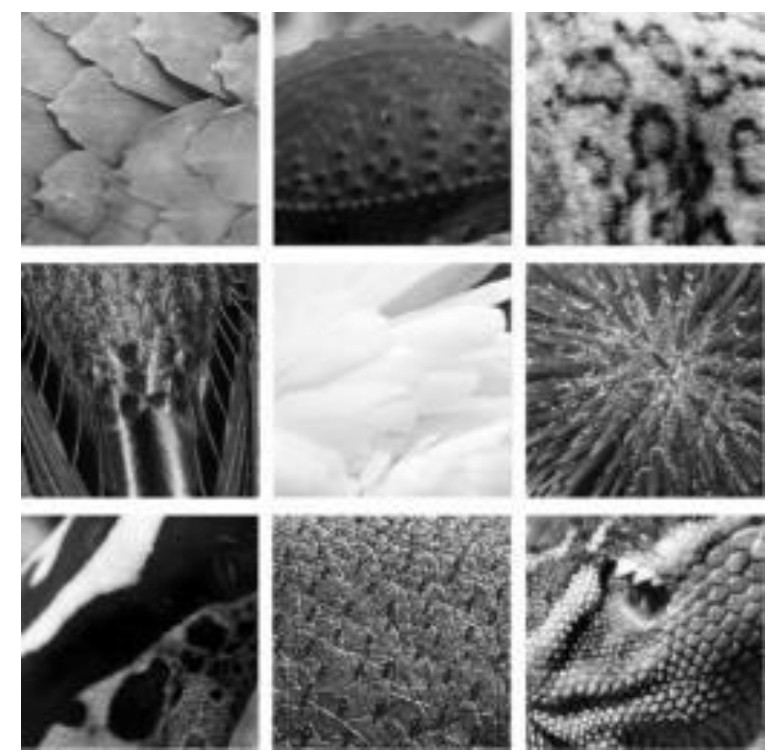

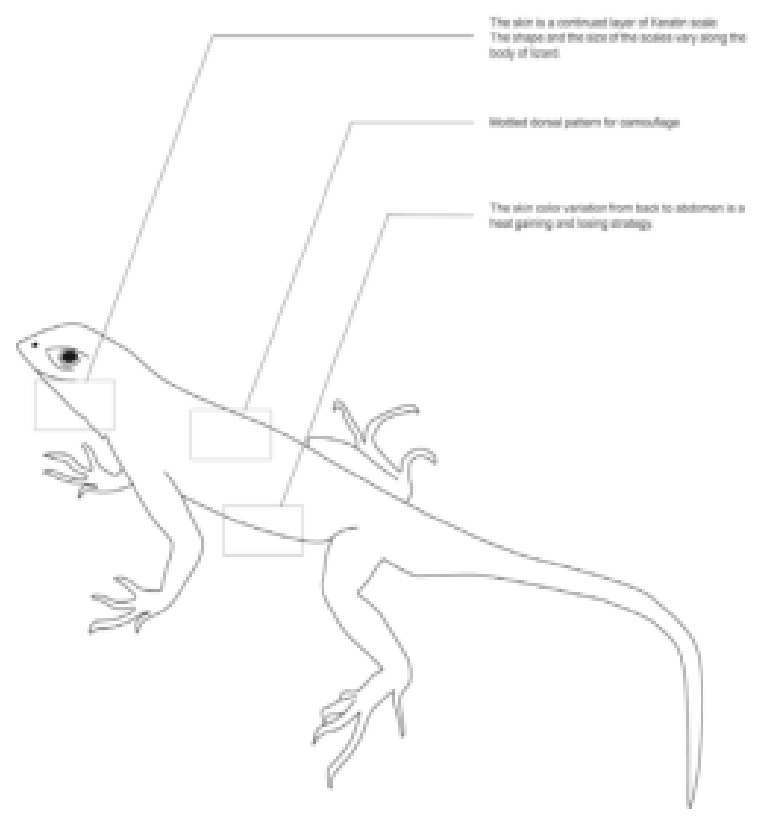

Figura 1: Ejemplo de inspiración biomimética para el control ambiental de un edificio basado en la piel de un lagarto. Fuente: Univ. USC Los Ángeles EU 2004.

En el año 2013 Mazzoleni (Mazzoleni, 2013) propone la biomimética para resolver estados de optimización y Granero (Granero, 2013), presentaba una investigación enumerando los antecedentes metodológicos y las herramientas de diseño en el proyecto arquitectónico de la envolvente biomimética. En la hipótesis de trabajo se parte de la afirmación: que el entorno construido es el resultado del proceso de mejoramiento del hábitat de la sociedad humana. La evolución del habitar del hombre ha experimentado modificaciones que se rigen por un mayor rendimiento físico y estético. Asimismo, dentro de la definición de biomimética, la arquitectura puede ser considerada como una forma de vida artificial, que está sujeta, al igual que el mundo natural, a los principios de la morfogénesis, de la codificación genética, de la replicación y de la selección. La creación de prototipos y el poder creativo de la evolución natural, se emulan mediante la creación de modelos virtuales de arquitectura que respondan a los cambios del entorno. El éxito de la evolución se estimula y se desarrolla. Los prototipos son las estructuras profundas, primitivas y simples, ligados por relaciones de proximidad entre otras que reaccionan a cambios externos al sistema y evolucionan o se desarrollan. 


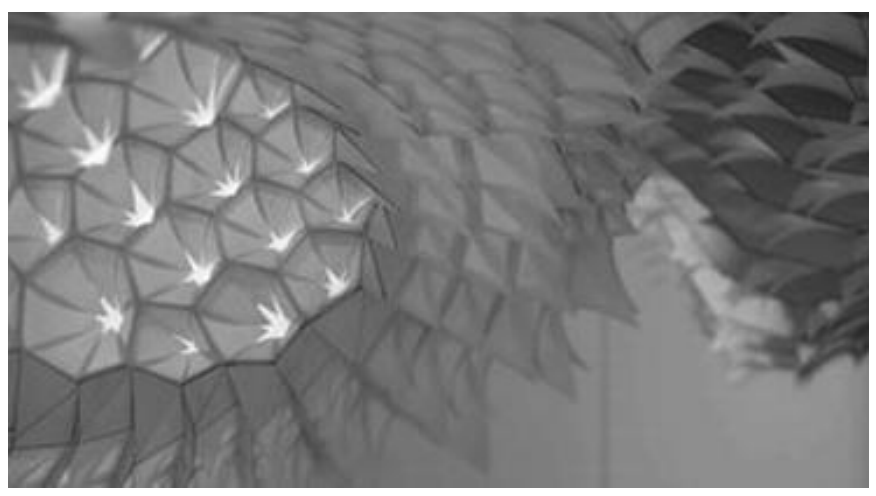

Figura 2: Higroscopio de Achim Menges y Steffen Reichert. Fuente: Menges y Reichert, 2012.

Achim Menges y Steffen Reichert en el año 2012 presentaron sus investigaciones en cuanto a la búsqueda de una nueva manera de materializar el límite del espacio arquitectónico. El trabajo exploró la inestabilidad dimensional de la madera en relación al contenido de humedad y como se emplea para construir un clima de respuesta morfológico. Patricia Muñoz ha experimentados desde la morfología en Diseño Industrial el "diálogo entre la morfología y la fabricación digital" prestando especial interés en las características físicas de los materiales y su adaptación a los cambios físicos sometidos manualmente. Ahora bien, en el contexto de Latino América hay que destacar que "el uso de técnicas emergentes en procesos de diseño son parte de exploraciones académicas y no de la práctica profesional cotidiana" (Herrera, 2014). Estimamos que es necesario revertir esta situación, e integrar las exploraciones académicas a la práctica profesional, siguiendo el ejemplo de USA y EU pero en el contexto latinoamericano. En la actualidad nos encontramos analizando investigaciones multidisciplinarias en publicaciones en revistas científicas y profesionales. La finalidad es que la comunidad de profesionales pueda seguir el desarrollo de una investigación que busca hallar un modelo optimo, un desarrollo autónomo, anticipando problemas de una práctica futura. La investigación se realiza sobre un mecanismo que permite que el sistema pueda mantenerse por sí mismo. Como señaláramos anteriormente y de acuerdo a Schumacher y a Bunge, para que la arquitectura se trasforme en un sistema material físico y dinámico necesita de un mecanismo. Podemos inferir que en la actualidad este mecanismo está relacionado a la eficiencia energética y la producción de energía, como elemento que produce cambios y ayuda en el mantenimiento del sistema por sí mismo, estos mecanismos convierten materia inorgánica en orgánica gracias a la luz o gracias a los vientos, que corresponden a fenómenos similares a la fotosíntesis (energía solar fotovoltaica) y al trabajo (energía cinética o energía eólica). En estos meses hemos trabajado en la etapa de simulación, modelando de la estructura profunda, primitiva y simple, modificando su morfología con la finalidad de optimizar el diseño y con la posibilidad de compartir el diseño para ser auto-producido por los profesionales-usuarios de impresoras 3D. Esta búsqueda está relacionada a la creación de mecanismos (Bunge) que permitan la "autopoiésis" (Schumacher).

\section{Un nuevo paso selectivo en la producción académica arquitectónica, el espacio topológico arquitectónico digital}

Las investigaciones tiene valor solo cuando podemos transferirlas a los talleres y a los alumnos, por este motivo además de fundamentar el proceso de morfogénesis a nivel teórico, se ha diseñado un procedimiento para inducir la construcción de un lenguaje arquitectónico propio, basado en el proceso selectivo, que permita llegar como resultado a la generación de superficies compleja y adaptada a partir de conocimientos previos y habilidades previas. Por todo lo expuesto anteriormente, entendemos que la estructura morfológica que permite la definición formal del límite del espacio vivible y que responde de manera adecuada a las demandas espaciales actuales, es el espacio topológico arquitectónico y su límite es una superficie topológica arquitectónica o una masa topológica arquitectónica. Este espacio que está influenciado por el concepto de convergencia, conectividad, continuidad, vecindad, etc. y que corresponde a la forma topológica arquitectónica; pertenece y a las demandas de cubiertas de espacios de grandes luces, es la que mejor se adapta.

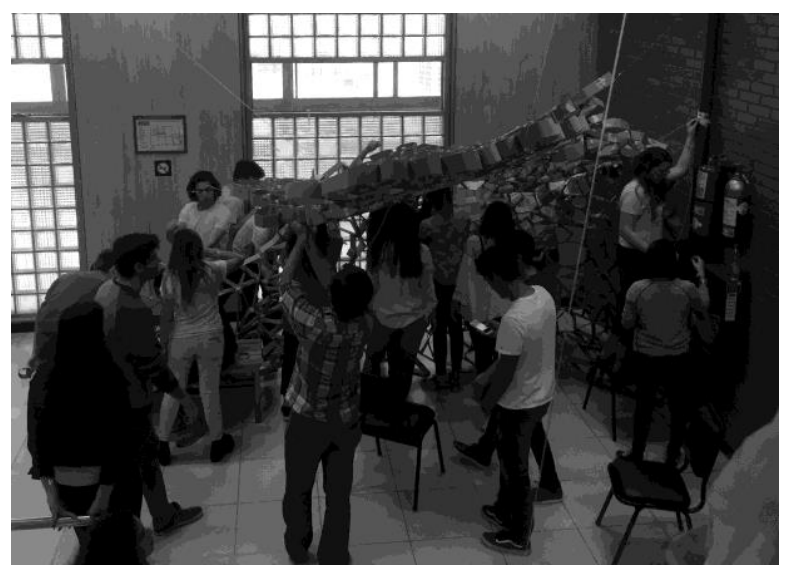

Figura 3: Ejemplo de concepción y construcción de los alumnos. 2016

Para los fines didáctico, este espacio es el pretexto que incentiva la actividad exploratoria e induce al descubrimiento de los componentes y las reglas que los relacionan. Podemos inferir que este espacio, es producto de una morfogénesis biyectiva con variables diferenciales, en una geometría diferencial, vinculado al análisis matemático y generador del espacio tetradimensional. Esta Morphogenesis (Matsuro Sasaki), Bio-Morfismo (Meredith Michael) o Arquitectura Biodigital o Genética (Estévez) la interpretamos como la generación de la frontera del espacio arquitectónico a partir de un prototipo inicial (genotipo, fenotipo o estructura profunda) y una familia de parámetros iniciales. 


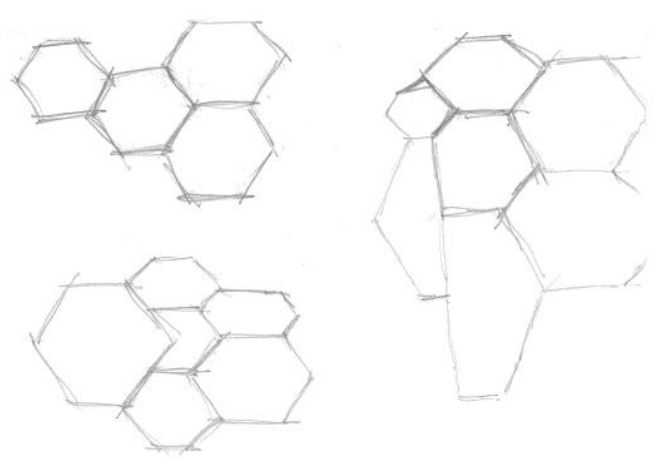

Figura 4: Ejemplo de concepción y construcción de los alumnos. 2016

Éstos últimos son los que sufre modificaciones para mantener las relaciones formales que guardan entre sí, los parámetros (a modo de PSO Optimización de Sistemas o Nube de Partículas por enjambre "Particle Swarm Optimization") son los que evolucionan de manera natural al igual que el vínculo que entre los prototipos iniciales. Es decir que la utilización de variables y algoritmos para generar un árbol de relaciones matemáticas y geométricas, nos permiten llegar al diseño y generar todo un rango de posibles soluciones; esto es posible gracias a la variabilidad de los parámetros iniciales. Pero las relaciones formales también son expuestas a variabilidad. Este proceso configurativo se fundamenta, en las transformaciones que se han puesto de manifiesto en el lenguaje para comunicar el sentido, la dialéctica de la arquitectura y que ha sido influenciado por el cambio evolutivo en la filosofía del lenguaje, el deconstructivismo (Heidegger). $Y$ en el caso específico de la forma de la arquitectura, esta evolución y selección se manifiesta en la fragmentación, en un diseño no lineal, en una geometría euclídea, con formas no rectilíneas, con una distorsión en la estructura y con la dislocación de la envolvente. También la forma del límite del espacio arquitectónico es sensible al principio de composicionalidad de Derrida, que toma aspectos vinculados con los diferentes significados de un texto y su vinculación con las palabras utilizadas. Sus diferentes significados son extraídos descomponiendo la estructura del lenguaje en el que está redactado. Tal es el caso de la apariencia visual, la estimulante impredicibilidad y el caos controlado de Eisenman y Libeskind. Un nuevo giro ha proyectado la gramática generativa de Chomsky, donde plantea que la adquisición del lenguaje es individual, parte de cuatro niveles o jerarquías que son: sin restricciones, sensible al contexto, libre de contexto y regulares.
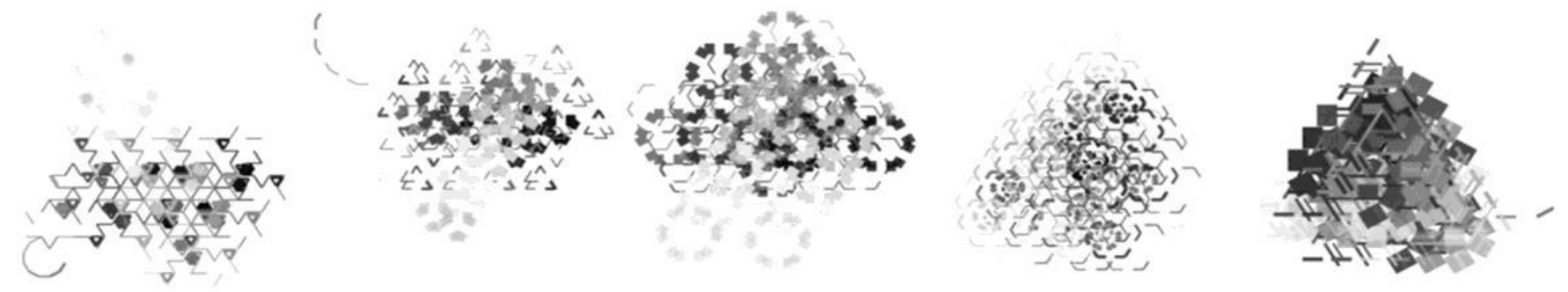

Figura 5: Concepto de evolución realizado por la autora con generador evolutivo de imágenes de Sistema-L

En esta evolución las formas surgen como el resultado de la relación entre parámetros constructivos, estructurales y ambientales, sugiriendo suaves transiciones con superficies curvas, con interpolaciones o gradientes y correlacionadas, emerge como una morfogenesis digital de crecimiento y diferenciación. Si retomamos el caso de las investigaciones sobre el comportamiento de los materiales de la Dra. Muñoz, el proceso de experimentación es de abajo hacia arriba, los funiculares de Gaudí o las investigaciones de Frei Otto. En otro extremo encontramos el diseño generativo de Smart Geometry Group con reglas bien definidas, con un orden preestablecido, con herramientas digitales y aplicadas a la arquitectura. Este último orden es establecido con parámetros vinculados al desarrollo del proyecto con rigurosidad programática. En este último, el lenguaje arquitectónico generativo y evolutivo, deriva de una metodología precisa vinculada al estudio de prototipos, establecimientos de reglas y definición de variables dan como resultado una arquitectura parametricista (Schumacher), dentro de la arquitectura digital y a la selección. Algunas aplicaciones de la representación digital como Grassopper o Dynamo son idóneas para el desarrollo de estas última investigaciones, aunque existen otras posibilidades que son aplicables desde el mismo software de modelado. Los conceptos de optimización y evolución establecen relaciones de continuidad con elementos celulares o a la inversa, la continuidad se ve interrumpida por un entorno intersticial patrones, rutas, reglas de teselación, etc. En este contexto los algoritmos de procesos referidos a la inteligencia de enjambre a base de procedimientos, optimizan el problema teniendo en cuenta posibles soluciones de posicionamientos de los objetos en el espacio, indudablemente no nos garantiza que la posición sugerida sea la mejor solución, pero la aplicación de los PSO en el proceso de diseño arquitectónico, nos proporciona un conjunto de óptimos posibles que son equivalentes en términos de diseño. De esta manera llegamos a un espacio arquitectónico tipológico diferencial, isomorfo cuyas funciones son continuas hasta los límites, dentro de una geometría diferencial, inmerso en una arquitectura paramétrica. Con un conjunto de elementos y relaciones, en el caso de las superficies concretas que delimitan el espacio arquitectónico, estos elementos al transformarse en elementos concretos dejan de ser flexibles, una vez determinada la forma de la envolvente, los parámetros de los elementos constitutivos se fijan por razones vinculadas a su tectonicidad, entre otras. Este congelamiento de los parámetros de la unidad elemental nos limita e invita a buscar el dinamismo propio (kinetic) de las estructuras con un comportamiento similar a una 
membrana en las relaciones, si bien en un artículo previo publicado en la RHS (2013), el autor se refiere a la interpretación del límite del espacio como una membrana cuyas cualidades de flexibilidad la hacen apropiada a las solicitaciones de tracción, en ese caso definíamos la membrana como dos superficies y un espacio entre ellas formando un todo. También se expuso que a los efectos de la investigación, los elementos son abstractos y se desarrollaban en un contexto puramente digital. El trabajo se basó en trabajos de matemáticos y en los teselados; en dicha oportunidad se partió de una célula o axioma y se replicó con características hereditarias, dando como resultado una endosimbiosis seriada. En la siguiente etapa y al querer transferir estos elementos al contexto real, se produjeron algunas modificaciones que se relacionan con la necesidad de que los cambios se provoquen en las relaciones más que en los elementos iniciales, pero además se introduce el concepto de relación como un nexo constituido por un nuevo elemento que sea vínculo y proporcione la continuidad y la eficacia, el mecanismo. Aunque los elementos iniciales sufran variaciones en sus parámetros, los cambios más relevantes deben aplicarse a los elementos que son vínculos y que constituyen la unión entre los elementos o prototipos iniciales. Estos vínculos deben ser relaciones adecuadas que sean capaces de soportar las tensiones (tensegridad) necesarias para que la configuración mantenga su forma tridimensional. Esta nueva etapa de la investigación nos traslada al análisis y clasificación de los distintos modos de vínculo y se relacionan con uniones, combinaciones, tipos y materiales.

\section{Metodología}

La metodología utilizada para esta investigación teórica, responde a una estrategia general de revisión teórica y de exploración de análisis de estudios descriptivos y concluyentes. Con Métodos inductivo, mixto con exploración y observación. El trabajo de investigación explora el espacio relativo, este espacio se genera a partir del diseño de la envolvente arquitectónica que desafía condiciones de rigidez con la estabilidad multidimensional y el equilibrio metabólico, retando la organización espacial de los límites arquitectónicos y su fundamento está dado en la aplicación de actividades de exploración morfológica, tectónica y tecnológica. Se introducen los conceptos vinculados a la geometría diferencial desde los instrumentos cotidianos del que hacer arquitectónico como la maqueta táctil, vinculando el concepto de prototipo al elemento inicial y las distintas relaciones.

\section{Desarrollo}

Para la investigación se gestiona una propuesta que tiene como punto de partida de la necesidad de modelar asociativamente y en segmentación una superficie que sirva como límite del espacio arquitectónico. Para ello, se establece que la experiencia se llevará a cabo integrando datos del mundo exterior con modelos digitales, a que los que se los pueda someter a la simulación y que sean factibles de ser realizados directamente desde producción digital. Ante los resultados obtenidos en las experiencias publicadas anteriormente, se reformula la actividad incitativa. La misma consta de una experimentación colaborativa modulada y se utiliza como pretexto para la introducción a la arquitectura topológica de grandes luces. Esta temática se encuadra en el área de la morfología. La experiencia consta de un ensayo incitativo activo, teatral y lúdico, cuyo objetivo es establecer una estrategia de abordaje (aplicación de PSO), para el diseño de las cubiertas para espacios de grandes luces. Este proceso permite un modelado paramétrico con el concepto de componente, familia o unidad básica y la conceptualización de actividades vinculadas a las relaciones tan presentes en los Algoritmos Evolutivos (EA). Se vinculan con la creación de superficies por semántica lógica y lingüística arquitectónica. Para el desarrollo de la experiencia se propone una figura geométrica bi-dimensional y se deberá materializar los segmentos que componen la figura con material rígido. Una vez seleccionada la figura se realizará una réplica de la unidad modificando alguno de los parámetros que facilite su relación con las figuras aledañas, obteniendo de esta manera una primera superficie que responde a los criterios establecidos. Como tarea final se propone el armado de una superficie con componentes cooperativos y en algunos casos colaborativos. Se deben negociar las asociaciones entre las distintas figuras creadas y establecer relaciones sostenidas en la integración. Finalmente la experiencia termina con el armado de una superficie única, que conforma el límite de un espacio con características propias y que es generador de instancias de cooperación y colaboración, donde se establecen reglas para la integración de cada unidad (figura) y aquí su relación a EA y PSO. La segunda parte de la experiencia, es la transferencia de la experiencia física y real a la generación de una superficie por computadora que tenga los mismos objetivos: límite de un espacio arquitectónico, las características del espacio arquitectónico y el módulo, componente, unidad básica o familia, que en este caso es tridimensional; las relaciones y negociaciones de las formas para constituir una superficie continua que sea la cubierta de un espacio de grandes luces y su sistema estructural y portante.

\section{Resultados}

Surge de la investigación, que el objetivo es crear una arquitectura que desafíe cualquier clasificación previa, con la exploración de niveles no vistos, con una resolución y una complejidad topológica inadvertida en lo que respecta al lenguaje de la arquitectura. Para ello se desarrollaron estrategias compositivas basadas en procesos puramente geométricos, pero físicos, táctiles, para establecer el delicado equilibrio entre lo esperado y lo imaginado; como resultado de la implementación de la geometría computacional numérica. La integración de técnicas digitales de modelado y fabricación de maquetas arquitectónicas, se interpreta como posible rampa que impulsa un proceso de generación espacial de la forma arquitectónica, con un código propio que vincula los componentes del espacio arquitectónico relativo materializando símbolos de la gráfica digital. Generando espacios de diálogo entre el análisis matemático y la geometría diferencial de superficies..

\section{Debate}

Los impactos previamente detectados con la aplicación de los sistemas digitales son: el aumento de la creatividad vinculada al diseño de los elementos del lenguaje, mayor libertad en el diseño de la envolvente arquitectónica y la versatilidad de la estructura del lenguaje arquitectónico. Existe un inminente 
límite físico entre lo que el diseño, como "pre-figuración" intelectual propone y su concreción física. Los descubrimientos presentados en el Higroscopio de Achim Menges y Steffen Reichert (2012) y las experiencias como S.C.A.L.E.S. (smart - continuous - active - layered environmental - system) propuesta en el libro de Mazzoleni (2013), manifiestan como interviene en la amplitud de la creación y de la imaginación. Esta nueva etapa de la investigación nos ha dirigido en la búsqueda de un elemento de arquitectura que pertenezca a al tetra-dimensión, que facilite la llegada a la adecuación absoluta de la envolvente del espacio arquitectónico en tiempo real.

\section{BIBLIOGRAFÍA}

BENYUS, J. (2002). Biomimecry. New York: HarperCollins e-books.

Frazer, J. (10 de 6 de 2012). Architectural Association School or Architectura. Obtenido de An Evolutionare Architecture: http://www.aaschool.ac.uk/publications/ea/intro.html

Iwamoto, L. (2009). Digital Fabrications: Architectural and Material Techniques. New York, USA: Princeton Architectural Press.

Moussavi, F. (2009). The function of Form. New York, USA: Acta and Harvard University Graduate School of Design.

Spuybrock, L. (2009). THE ARCHITECTURE OF VARIATION. New York, USA: Thames \& Hudson Inc.

\section{TRABAJOS CITADOS}

Muñoz, P. e. (2013). Diálogos entre morfología y fabricación digital. Buenos Aires, Argentina: De la forma.

Estévez, A. (1-5 de 12 de 2008). Arquitectura Biodigital. Recuperado el 30 de 12 de 2014, de Comunicades: http://cumincades.scix.net/cgibin/works/Show?_id=sigradi2008_172\&sort=DEFAULT\&search= Estevez\&hits $=2$

Bueno, E. (3 de 1 de 2008). Consideraciones y recursos para la concepción de la forma en la arquitectura de la era digital. Obtenido de Unicamp: http://www.fec.unicamp.br/ parc/vol1/n3/vol1-n3-bueno.pdf

Schumacher, P. (1 de 6 de 2011). La Autopoesis de la Arquitectura. Recuperado el 30 de 12 de 2014, de Universidad de Chile: http://www.revistas.uchile.cl/index.php/RA/issue/view/2430

Mazzoleni. (2013). Architecture Follows Nature-Biomimetic Principles of Innovative Design. En Architecture Follows Nature-Biomimetic Principles of Innovative Design. New York, New York, USA: CRC Press Series in Biomimrtics.

Granero, A. (2013). Antecedentes metodológicos y herramientas de diseño arquitectónico de la envolvente biomimética. Revista Habitat Sustentable , 3 (2), 10.

Bunge, M. (2012). Tratado de Filosifía-Ontología 2-Un Mundo de Sistemas (Vol. IV). Barcelona, España: GEDISA.

Herrera, P. (2014). Más allá de la ilusión de objetividad y/o subjetividad en los procesos de diseño. De Arquitectura , 27, 78. 